\title{
A representação descritiva no contexto da web semântica'
}

\author{
Descriptive representation in the semantic web context
}

Maria Elisabete CATARINO²

Terezinha Batista de SOUZA²

\section{Resumo}

Este artigo contém o resultado de uma pesquisa bibliográfica, cujo objetivo foi verificar como as práticas da representação descritiva estão relacionadas com a proposta de organização dos recursos da Web no contexto da Web Semântica. A pesquisa foi desenvolvida a partir de duas temáticas: uma que abarcou os conceitos referentes à Web Semântica, com o objetivo de compreender os procedimentos e as tecnologias a eles relacionados; outra que focou a representação descritiva, com o intuito de entender como a práxis da catalogação poderá auxiliar na inserção dos dados bibliográficos no contexto da Web Semântica, bem como averiguar ações que estão sendo realizadas nesse sentido. O levantamento foi feito nos idiomas português e inglês, em periódicos e livros das áreas de Ciência da Informação, Internet e Web, bem como em documentos disponíveis no site do Word Wide Web Consortium. O texto discorre sobre a Web Semântica e os conceitos básicos a ela relacionados: dados lincados, vocabulários, busca, inferência e aplicações verticais. Destaca o Resource Description Framework como modelo de descrição de recursos, que é o fundamento da Web Semântica. Com base na análise dos textos, nos exemplos apontados de ações que estão sendo desenvolvidas por algumas instituições, tais como Library of Congress, Dublin Core Metadata Initiative e Joint Steering Committee, e tendo como foco os objetivos acima explicitados, pôde-se inferir que as práticas da representação descritiva estão relacionadas com a Web Semântica na medida em que poderão ser aplicadas para tornar os dados bibliográficos em dados lincados da Web.

Palavras-chave: Dublin Core. Metadados. Representação descritiva. FRBR. RDA. RDF. Web semântica.

\begin{abstract}
This article presents the result of a bibliographic research developed with aim of verifying how the practices of descriptive representation are related to the proposal of Web resources organization in the Semantic Web context. The study was developed based on two main themes. The first involved concepts related to the Semantic Web with objectives of understanding which are the procedures and technology related to Semantic Web. The second concerned descriptive representation in order to understand how cataloging practices can be used to help bibliographic data insertion into the Data Web as well as to verify the actions that are being to developed in this direction. The research was developed in Portuguese and English, in papers and books in the information science area as well as in documents available in the site of the Word Wide Web Consortium. This article is based on Semantic Web and its concepts such as: linked data, vocabulary, query, inference and vertical applications. These results highlight the Resource Description Framework that is a resource description model based on Semantic Web. Based on text analysis, in examples of actions pointed out, which are being developed by some institutions, such as: Library of Congress, Dublin Core Metadata Initiative and Joint Steering Committee; and with focus on the objectives already mentioned, it can be inferred that the practices of descriptive representation are related to the Semantic Web, to the extent to which they can be applied to transform the bibliographic data into data linked to the Web.
\end{abstract}

Keywords: Dublin Core. Metadata. Descriptive representation. FRBR. RDA. RDF. Semantic web.

\footnotetext{
1 Artigo elaborado a partir da pesquisa em andamento intitulada "Contribuições da Ciência da Informação para Web Semântica", coordenada por M.E. CATARINO. Apoio financeiro do MCT/CNPq, Edital Universal 2010, processo 474215/2010-5.

2 Professoras Doutoras, Universidade Estadual de Londrina, Departamento de Ciência da Informação. Rod. Celso Garcia Cid, Km 380, Campus Universitário, Caixa Postal 6001, 86051-990, Londrina, PR, Brasil. Correspondência para/Correspondence to: M.E. CATARINO. E-mail: <beteca@uel.br>.

Recebido em 21/1/2012, reapresentado em 30/3/2012 e aceito para publicação em 25/4/2012.
} 


\section{Introdução}

O estudo apresentado neste artigo inicialmente enfatizava as questões da representação temática. Todavia, com o desenvolvimento da investigação e consequentemente maior compreensão do que é a Web Semântica e qual o relacionamento ou contribuições da Ciência da Informação para esse cenário, percebeu-se que também, e inclusive, a representação descritiva deveria ser estudada como contribuição para o desenvolvimento da organização da informação na Web.

Desde o início da pesquisa, havia o pressuposto de que a Ciência da Informação, mais especificamente a área de organização da informação, contribuiria com a Web Semântica devido à convicção de que a práxis de ambas são compatíveis. Neste texto, o foco está sobre a área da representação descritiva, pois o alicerce da Web Semântica está num modelo de descrição dos recursos da Web, o Resource Description Framework (RDF). Ao abordar a relação entre as práticas da representação descritiva e da Web Semântica, vislumbra-se que a atuação do profissional da informação na organização dos recursos da Web permitirá o compartilhamento e a preservação dos dados bibliográficos (na Internet), conforme prescrevem as regras da catalogação.

O termo Web Semântica foi criado para representar o projeto do Word Wide Web Consortium (W3C), que pretende estruturar as páginas da Web, dando a elas significado, desenvolvendo um ambiente onde agentes de software possam criar sofisticadas tarefas para os usuários a partir de conteúdos de várias páginas e bases de dados ${ }^{3}$ da Web (Berners-Lee et al., 2001). A estruturação desses conteúdos ocorre por meio de normas e regras para a criação de armazéns de dados na Web, construção de vocabulários e manipulação de dados (Word Wide Web Consortium, 2010).

Ao se pensar em estruturação dos conteúdos, logo se pode fazer uma analogia com a área de organização da informação, especialmente a representação descritiva e temática. Nesse contexto, entender quais regras e normas estão sendo adotadas pelos desenvolvedores da Web e como a práxis da representação descritiva está se desenvolvendo nessa área é imprescindível. Este artigo apresenta o resultado de um estudo feito a partir de uma revisão bibliográfica sobre os conceitos da Web Semântica e da representação descritiva, bem como as ações que já estão em andamento para tornar os dados bibliográficos dados lincados da Web.

Com o objetivo de verificar como as práticas da representação descritiva estão relacionadas com a proposta de organização dos recursos da Web, no contexto da Web Semântica, a pesquisa bibliográfica foi realizada a partir de duas temáticas. O primeiro tema abarcou o conceito Web Semântica e outros a ele relacionados (Dados Lincados, RDF e Web de Dados) com o objetivo de compreender as tecnologias e os procedimentos necessários para a implantação da Web Semântica. O outro tema focou a representação descritiva com o intuito de entender como a sua práxis poderá auxiliar na inserção dos dados bibliográficos na Web Semântica, bem como averiguar ações que têm sido realizadas neste sentido.

A pesquisa foi realizada nos termos acima citados, nos idiomas português e inglês, em periódicos e livros das áreas de Ciência da Informação, Internet e Web. Também foram consultados os documentos disponíveis no site do W3C.

Com base na leitura dos textos e tendo como foco os objetivos explicitados, procedeu-se a uma análise para averiguar o pressuposto de que a prática da representação descritiva está relacionada com a da Web Semântica e, mais especificamente, como os dados bibliográficos poderão tornar-se dados lincados.

Para discorrer sobre essa perspectiva, apresentamos, inicialmente, os conceitos relativos à Web Semântica e as tecnologias a ela relacionadas, definições da representação descritiva e novas perspectivas da catalogação no cenário da Web.

\section{Web semântica}

O conceito da Web Semântica ${ }^{4}$ foi referido em 2001, na publicação de um artigo na revista Scientific American,

\footnotetext{
3 Neste texto considera-se a bases de dados no sentido de qualquer conjunto de registros relacionados entre si, conforme define Sawaya (1999, p.112) "Conjunto de informações relacionadas entre si, referentes a um mesmo assunto e organizadas de maneira útil, com o propósito de servir de base para que o usuário recupere informações".

${ }^{4}$ No entanto, Berners-Lee considerou que o título mais adequado para o projeto seria Data Web ou Web of Data (Web de Dados) (King, 2007; Word Wide Web Consortium, 2010). Optou-se neste texto por padronizar a denominação do projeto, utilizando apenas o termo Web Semântica para que haja maior entendimento do conteúdo.
} 
por Tim Berners-Lee, James Hendler e Ora Lassila. Os autores explicam que não é uma Web separada, mas uma extensão da atual, na qual a informação é dada com um significado bem definido, permitindo melhor interação entre computadores e pessoas (Berners-Lee et al., 2001). Trata-se de uma nova geração da Web, projeto do W3C, cujo intento é desenvolver tecnologias, linguagens, padrões e recomendações que tornem a informação legível pelas máquinas.

Segundo Berners-Lee et al. (2001), os computadores necessitam ter acesso a coleções estruturadas de informações (dados e metadados) e a conjunto de regras de inferência que ajudem no processo de dedução automática para que seja aplicado o raciocínio automatizado.

A Web Semântica é composta por alguns elementos: uma filosofia, um conjunto de princípios para design, grupos de trabalho e tecnologias para a implantação desses princípios e propostas. Alguns elementos são expressos em especificações formais que incluem o modelo RDF, formatos de intercâmbio de dados (como por exemplo: RDF/XML, N3, Turtle, N-Triples) e notações, tais como RDF Schema (RDFS) e a Web Ontology Language $(\mathrm{OWL})$, para prover uma descrição formal dos recursos e dos conceitos, termos e seus relacionamentos, num específico domínio do conhecimento (Word Wide Web Consortium, 2001).

O W3C pretende implementar, divulgar e promover linguagens, normas e recomendações que permitam que os computadores possam, a partir de dados dispersos em sites e bases de dados da Web, compor interações na rede e gerar serviços e produtos. O termo Web Semântica representa uma Web de dados lincados (linked data). As tecnologias recomendadas pelo W3C permitirão que as pessoas e as instituições criem armazém de dados, construam vocabulários e escrevam regras para a manipulação dos dados (Word Wide Web Consortium, 2010).

Os conceitos básicos que compõem a ideia da Web Semântica (dados lincados, vocabulários, busca, inferência e aplicações verticais) serão apresentados a seguir.

\section{Dados lincados}

Conforme o Word Wide Web Consortium (2010), para tornar a Web Semântica uma realidade, é importante ter uma grande quantidade de dados disponível em um formato padrão, acessível e gerenciável por tecnologias apropriadas. Além do acesso aos dados da Web, é necessário, ainda, criar relações entre os dados. Dados lincados é o nome que se dá às coleções de dados relacionados na Web.

Para a publicação e vinculação dos dados, é preciso adotar o modelo recomendado pelo W3C, o RDF, que é o alicerce da Web Semântica e um modelo padrão para intercâmbio de dados na Web (RDF Working Group, 2004). As tecnologias disponíveis devem ter como fundamento o modelo RDF para possibilitar o acesso aos dados existentes em bases de dados, sejam elas relacionais (estruturadas em tabelas e seus relacionamentos) ou outras, como, por exemplo, as que estão estruturadas na forma de linguagens de marcação (XML ou HMTL) (Word Wide Web Consortium, 2010). OW3C recomenda: RDF; Gleaning Resource Descriptions from Dialects of Languages (GRDDL) que é uma ferramenta desenvolvida pela W3C para a obtenção de dados RDF a partir de documentos XML e em particular XHTML (Halpin; Davis, 2007); Protocolfor Web Description Resources (POWDER), um protocolo para descrição e localização de recursos da Web que ajuda o usuário a decidir se um determinado recurso é de interesse (Scheppe, 2009); Resource Description Framework Atribute (RDFa) que descreve o conteúdo de uma informação utilizando atributos das tags de linguagens de marcação como Extensible Hypertext Markup Language (XHTML) (Adida; Birbeck, 2008); Relational Databases to RDF Mapping Language (R2RML), uma linguagem para mapear dados de bases de dados relacionais para bases de dados RDF (Das et al., 2010); Rule Interchange Format (RIF) padrão desenvolvido para facilitar integração de conjunto de regras e é composto por um conjunto interconectado de dialetos representando regras de linguagens com diferentes características (Morgenstern et al., 2010); e Protocol and RDF Query Language (SPARQL), uma linguagem de busca padronizada proposta pelo RDF Data Access Working Group (DAWG) do W3C, considerada uma chave para a Web Semântica (Prud'Hommeaux; Seaborne, 2008; Word Wide Web Consortium, 2010).

Um exemplo de dados lincados, citado pelo W3C, é a DBPedia (http://wiki.dbpedia.org) cujo objetivo é extrair informação estruturada da Wikipedia e torná-la disponível 
na Web, permitindo executar consultas complexas lincando os dados da Wikipedia com outros conjuntos de dados da Web (Hahn et al., 2010), essencialmente reformata o conteúdo da Wikipedia disponível para RDF. A importância da DBPedia não está somente em incluir conteúdos da Wikipedia, mas também incorporar links de outras bases de dados, como, por exemplo, o Geonames (http://www. geonames.org) que é uma base de dados de nomes geográficos que contém mais de dez milhões de nomes geográficos, além de informações gerais e estatísticas sobre países, cidades etc. Ao fornecer esses links extras (em RDF), as aplicações podem explorar o conhecimento de outras bases de dados e consequentemente fornecer ao usuário um resultado bem mais completo.

\section{Vocabulários}

Nomeadamente, na Web Semântica, os vocabulários são constituídos por termos que representam conceitos, possíveis relacionamentos entre eles e as limitações no seu uso. Os vocabulários podem também ser denominados "ontologias". Segundo o Word Wide Web Consortium (2010), na prática, não existe uma recomendação para o uso de um ou outro conceito, mas, comumente, a tendência é usar o conceito "vocabulário" para coleções de termos mais simples e "ontologia" para as coleções mais complexas 5 .

Como exemplo da utilização de vocabulários para a Web Semântica, cita-se um destacado pelo Word Wide Web Consortium (2010): para a área da saúde - os profissionais médicos podem usar vocabulários para representar informações sobre os sintomas, doenças e tratamentos; já as empresas farmacêuticas, para representar informações sobre medicamentos, dosagens e reações. A combinação dessas informações com os dados sobre os pacientes permitiria uma ampla gama de aplicações inteligentes: ferramentas de apoio à decisão sobre possíveis tratamentos; sistemas de monitoramento da eficácia das drogas nos tratamentos e seus efeitos colaterais; ferramentas de apoio à investigação epidemiológica etc.

O W3C oferece várias possibilidades para definir vocabulários: RDF e RDF Schema, Simple Knowledge
Organization System (SKOS), OWL e RIF. A escolha da tecnologia vai depender da complexidade exigida pela aplicação na qual o vocabulário será utilizado.

\section{Busca}

No contexto da Web Semântica, o termo busca (Query) é usado genericamente para se referir às tecnologias e aos protocolos desenvolvidos para a recuperação da informação (Word Wide Web Consortium, 2010).

Assim como as bases de dados tradicionais necessitam de linguagens específicas de consulta (por exemplo, a SQL para as bases de dados relacionais, e o XQuery para as bases de dados em XML), a Web Semântica, com seus dados formatados em RDF, necessita de uma linguagem própria, no caso, a SPARQL. Essa linguagem permite fazer buscas e receber os resultados em Hyper Text Tranfer Protocol (HTTP) ou Simple Object Access Protocol (SOAP).

\section{Inferência}

Na lógica, inferência é definida como:

O processo de concluir uma afirmação a partir de outras afirmações. Por exemplo, com base nas afirmações 'Deus existe' e 'Se Deus existe, a felicidade eterna é possível', pode-se inferir 'A felicidade eterna é possível'. Um ARGUMENTO é uma inferência, usada para efeitos de persuasão racional (Murcho, 2011, online).

Na Web Semântica, os dados são modelados como um conjunto de relações entre os recursos da Web. Nesse contexto, "inferência" refere-se à descoberta de novas relações entre os recursos Web com base nos dados e em algumas informações adicionais sob a forma de um vocabulário ou conjunto de regras (Word Wide Web Consortium, 2010).

Ambas as formas de informações adicionais recorrem a técnicas de representação do conhecimento. A primeira forma (os vocabulários) define classes e subclasses de conceitos, e, ainda, como cada recurso pode ser associado a essas classes, caracterizando as relações entre as classes e suas instâncias.

\footnotetext{
${ }^{5}$ O conceito de complexidade usado aqui é no sentido de grau de formalidade, como, por exemplo, no âmbito das ontologias que, segundo a tipologia de Uschold e Gruninger, vão desde as Altamente Informais (as mais simples), até as Rigorosamente Formais (as mais complexas) (Catarino, 2009).
}

TransInformação, Campinas, 24(2):77-90, maio/ago., 2012 
A segunda forma (as regras) concentra-se na definição de um mecanismo geral de descoberta e geração de novas relações baseadas nas já existentes, bem como em programas lógicos, como, por exemplo, Prolog.

As ferramentas recomendadas pelo Word Wide Web Consortium (2010), para definir vocabulários são: RDFS, OWL ou SKOS. Para as abordagens baseadas em regras, o W3C recomenda o padrão RIF.

\section{Aplicações verticais}

Aplicações verticais (vertical applications) é uma expressão usada pelo Word Wide Web Consortium (2010), para se referir às aplicações genéricas desenvolvidas por comunidades específicas que exploram as tecnologias do W3C. Algumas dessas áreas de aplicação podem formar grupos de trabalho no W3C, colaborando com os demais membros do consórcio para explorar novas possibilidades para a Web Semântica. Esses grupos trazem importantíssimas contribuições para o aperfeiçoamento das recomendações, tecnologias e padrões desenvolvidos pelow3C.

O W3C cita vários exemplos, destacando-se o da área da Saúde e Ciências da Vida: The Health Care and Life Science Interest Group, que foi criado, em 2005, para explorar a usabilidade de tecnologias de Web Semântica na área, como a divulgação do conhecimento científico, processos de aprovação de medicamentos, entre outros. O grupo tem produzido várias manifestações e publicações, tem organizado workshops e conferências, e conseguiu transformar essa área de aplicação em uma das comunidades mais ativas de usuários das tecnologias da Web Semântica.

\section{RDF: o fundamento da web semântica}

Apresentados os conceitos básicos da Web Semântica, passamos ao RDF, considerado o fundamento da Web Semântica e que está relacionado com a representação descritiva dos recursos da Web. Segundo Allemang e Hendler (2011, p.27), "RDF, RDFS e OWL são as linguagens básicas de representação da Web Semântica".
RDF é um modelo para a descrição de recursos. Allemang e Hendler (2011, p.49) o definem como um "sistema de modelagem de dados". Segundo Manola e Miller (2004), RDF é uma forma de representação dos metadados dos recursos Web, tais como título, autor, data de modificação de uma página da Web, copyright e informações sobre licenças de documentos Web ou informações sobre o compartilhamento.

Nesse modelo de descrição de dados, existe uma estrutura bastante simples. Os recursos Web, ou seja, todas as coisas na Web, podem ser descritos em triplos: Subject, Predicate, Object (Allemang; Hendler, 2011). Ainda nesse sentido, Manola e Miller (2004) dizem que o RDF é baseado na identificação de recursos por meio de identificadores da Web, denominados Uniform Resource Identifiers (URI), e na descrição dos recursos em termos de propriedades, ou seja, atributos que descrevem um recurso ou as características que podem identificar um recurso e seus valores correspondentes. Para exemplificar os conceitos do modelo RDF (Subject/Recurso, Predicate/Propriedade, Object/Nalor), a seguir serão apresentados alguns exemplos de descrição de dados no modelo RDF.

Conforme Manola e Miller (2004), no site do W3C, se quisermos especificar o autor (Creator) ao descrever um dado recurso, teríamos a seguinte declaração:

http://www.example.org/index.html has a creator whose value is John Smith.

No exemplo acima, o triplo seria:

Subject: o site - http://www.example.org/ index.html

Predicate: a propriedade - creator

Object: o valor correspondente à propriedade: John Smith

Essa estrutura permite uma forma bastante simples de mesclagem de dados (merging data). Para Allemberg e Hendler (2011), não é necessário estruturar os elementos de metadados em forma de tabelas/colunas para poder mesclar ou recuperar os dados. A mescla dos dados é, então, transformada, a partir dos triplos, numa simples questão de reunir todas as declarações ${ }^{6}$ oriundas de diversas fontes num único lugar.

\footnotetext{
6 Declaração pode ser definida como sendo uma parte dos metadados que descreve um recurso na Web. Composta de um "Subject", um "Predicate" e um "Object", ou seja, de um Recurso, uma Propriedade e seu Valor correspondente: "Moby Dick/has author/Herman Melville" é uma declaração. Na proposta da Web Semântica, não há um registro de metadados, mas um grupo de declarações relacionadas. As declarações relacionadas expressam a mesma descrição que um registro de metadados expressaria em outros sistemas (Word Wide Web Consortium, 2011).
} 
Essa simples estrutura dos triplos tem um desafio (que não é apenas do RDF, mas sim de toda a Web): um identificador (identifier) único para cada recurso disponível. Na Web, a solução é o URI, adotada também pelo RDF.

Considerando que o conceito básico da RDF é o de que tudo seja identificado por meio de URI, como uma forma adequada para que as declarações em RDF e RDF/XML sejam processáveis por máquina, nesse sentido, a declaração do exemplo anterior seria da seguinte forma:

Subject: o site - http://www.example.org/index. html

Predicate: a propriedade creator - http://purl. org/dc/elements/1.1/creator

Object: o valor correspondente à propriedade http://www.example.org/staffid/85740

Nota-se que tanto a propriedade quanto o seu valor correspondente foram representados em seus respectivos namespaces que é uma coleção de nomes (ou termos) usados em documentos XML como tipos de elementos (propriedades) e nomes de atributos (valores) (Word Wide Web Consortium, 2003) (Figura 1). A propriedade Creator faz parte do conjunto de termos do Dublin Core Metadata Elements (http://purl.org/dc/ elements/1.1/creator) e o valor correspondente possui um namespace específico (identificadores numéricos para o staff do refe-rido exemplo: http://www.example.org/ staffid/85740).

Essa declaração (Figura 1) seria representada, em RDF/XML, da seguinte forma: versão XML usada; indicação dos namespaces utilizados (no caso: RDF, DC e EXTERMS) e

82

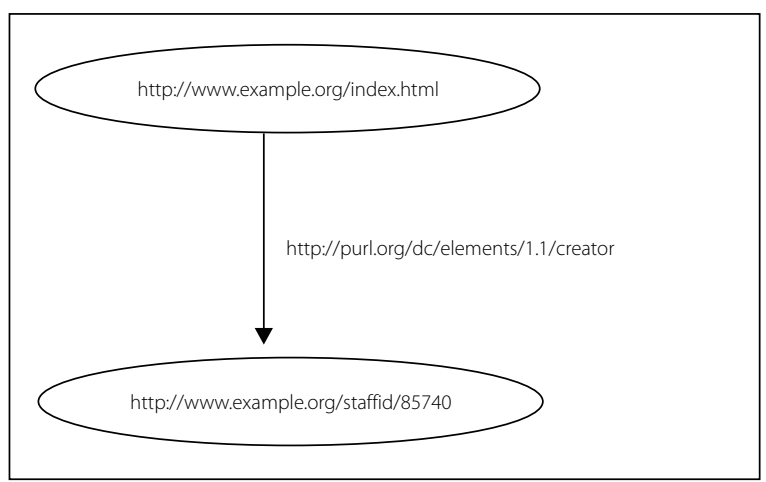

Figura 1. Uma declaração em RDF.

Fonte: Manola e Miller (2004). na sequência a descrição propriamente dita (Manola; Miller, 2004).

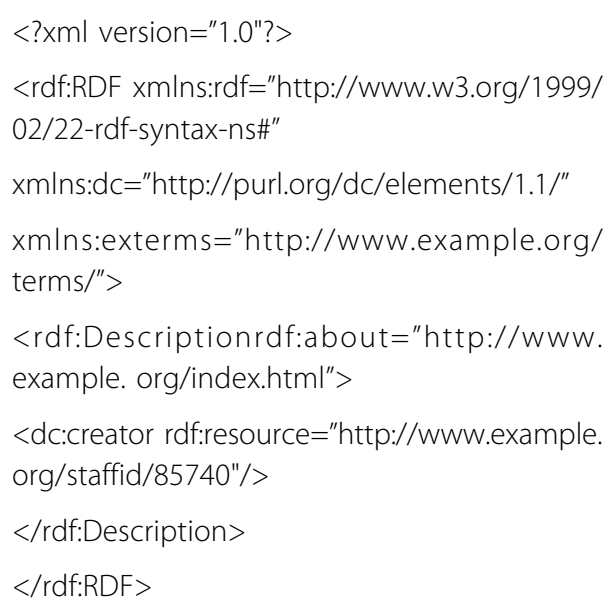

Deve-se ressaltar que a descrição de um recurso poderá ser composta, ou seja, a um Subject poderá relacionar-se mais de um Predicate e seus correspondentes Object (Figura 2). No exemplo utilizado, a declaração é referente ao Creator (autor) de uma página da Web (exemplo). No entanto, poderíamos ainda complementar a descrição com outras propriedades, tais como a data de criação e o idioma.

Subject: o site - http://www.example.org/ index.html

Predicate: a propriedade creator - http:// purl.org/dc/elements/1.1/creator

Object: o valor correspondente à propriedade: http://www.example.org/staffid/85740

Predicate: a propriedade creation-date - http:// www.example.org/terms/creation-date

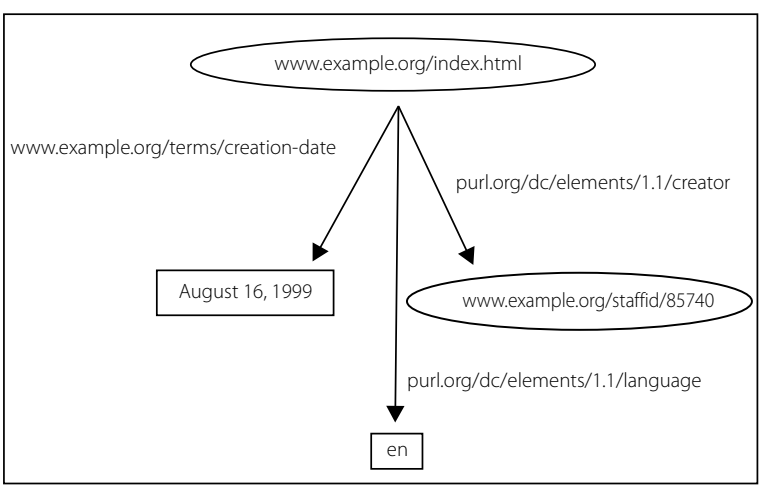

Figura 2. Descrição composta por várias propriedades. Fonte: Manola e Miller (2004). 
Object: o valor correspondente à propriedade: August, 16, 1999

Predicate: a propriedade language - http://purl. org/dc/elements/1.1/language

Object: o valor correspondente à propriedade: en

A Figura 2 mostra o gráfico dessa descrição.

Em RDF/XML, numa forma adaptada de Manola e Miller (2004):

$$
\begin{aligned}
& <\text { ?xml version="1.0"?> } \\
& <\text { rdf:RDF xmlns:rdf="http://www.w3.org/1999/ }
\end{aligned}
$$
02/22-rdf-syntax-ns\#"

xmlns:dc="http://purl.org/dc/elements/1.1/"

xmlns:exterms="http://www.example.org/ terms/">

$<$ rdf:Descriptionrdf:about="http://www. example.org/index.html">

$<$ dc:creator rdf:resource="http://www.example. org/staffid/85740"/>

$</$ rdf:Description $>$

$<$ rdf:Descriptionrdf:about="http://www. example.org/index.html">

<exterms:creation-date>August 16, 1999</ exterms:creation-date>

$</$ rdf:Description $>$

$<$ rdf:Descriptionrdf:about="http://www example.org/index.html">

$<\mathrm{dc}$ :language $>$ en $</$ dc:language $>$

$</$ rdf:Description $>$

$</$ rdf:RDF $>$

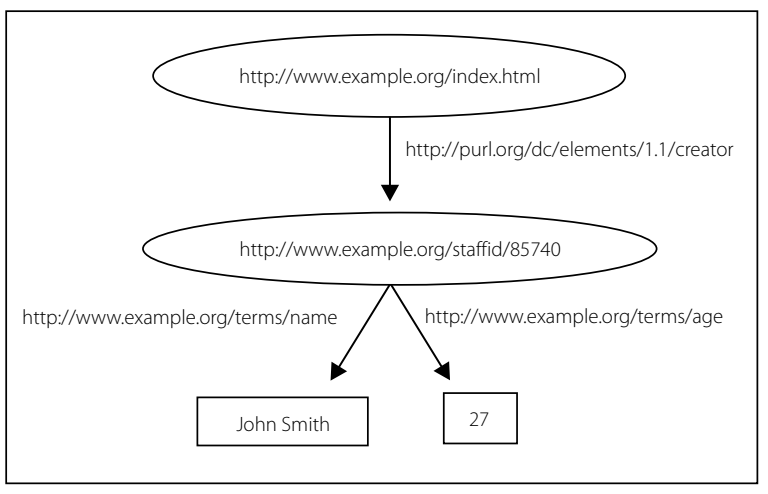

Figura 3. Descrição composta.

Fonte: Manola e Miller (2004).
O exemplo representa uma declaração simples, um recurso e suas propriedades. Contudo, as descrições no modelo RDF podem ser mais complexas. Por exemplo, um valor Object poderá ser ele próprio um Subject com suas propriedades Predicate e seus res-pectivos valores (Figura 3).

O intuito deste texto é apresentar a ideia RDF, com seus princípios básicos, no entanto, ainda existem muitas outras questões importantes que permeiam a descrição por meio do RDF. Uma dessas questões é o fato de um valor poder ser representado tanto por um URI (no exemplo http://www.example.org/staffid/85740 - URI referente ao Creator da página exemplo: John Smith) quanto por um string, ou seja, por um conjunto de caracteres alfanuméricos que podem formar dados, sejam eles palavras e/ou frases ou quaisquer outros tipos de dados. Conforme Sawaya (1999, p.450), string é "sequência conectada de caracteres ou de bits tratados como um só item de dados". No exemplo, os valores "John Smith" e "27" são strings que representam os valores das propriedades "name" $\mathrm{e}$ "age", respectivamente.

Um slogan que se refere à Web é "Anyone can say Anything about Anytopic (AAA)"(Allemberg; Hendler, 2011, p.11), ou seja, Qualquer um pode dizer Qualquer coisa sobre Qualquer assunto (QQQ). RDF está de acordo com esse slogan porque permite que qualquer fonte se refira a recursos em quaisquer namespaces. Mesmo um único triplo (subject/predicate/object) pode se referir a recursos em vários namespaces.

Como um modelo de dados, RDF fornece uma clara especificação do que tem que ser feito para mesclar informações de múltiplas fontes. RDF por si só não fornece algoritmos ou tecnologia para implementar esses processos. As tecnologias necessárias são: RDF Parser/ Serializer; RDF Store; RDF Query Engine; Application (Allemberg; Hendler, 2011). Neste texto, não serão abordadas as questões referentes a essas tecnologias; o objetivo é fazer uma análise de como essa proposta de organização dos recursos da Web, no contexto da Web Semântica, que tem como base o RDF, está relacionada com as práticas da representação descritiva abordada na Ciência da Informação.

Nesse sentido, serão abordadas questões a respeito da representação descritiva e, na sequência, sua relação com a Web Semântica. 


\section{Representação descritiva}

A representação da informação registrada tem como principal objetivo proporcionar a comunicação entre os documentos e os usuários da informação. Pode ser subdividida em representação descritiva e representação temática. A descritiva representa as características específicas do documento que permitem sua individualização e também define e padroniza os pontos de acesso responsáveis pela busca e recuperação da informação e pela reunião de documentos semelhantes. A temática detém-se na atribuição de assuntos aos documentos a partir da classificação bibliográfica, da indexação e da elaboração de resumos, facilitando a recuperação de materiais relevantes que dizem respeito a temas semelhantes.

Embora ambas sejam complementares, neste artigo, serão abordadas apenas as questões relativas à representação descritiva, uma vez que ela:

[...] refere-se aos aspectos da descrição formal dos documentos, o que inclui a descrição física e a descrição dos elementos para identificação dos mesmos; a atividade de representação descritiva é também chamada de catalogação (ou, mais especificamente, catalogação descritiva) em especial entre a comunidade de bibliotecas, e de descrição bibliográfica entre a comunidade dos serviços de informação científica (Ortega, 2011, p.45).

O trabalho de representação descritiva depara-se, muitas vezes, com questões relativas a diferentes documentos que precisam de instrumentos normativos, claros, abrangentes, cujos exemplos são a $2^{a}$ edição da Anglo-American Cataloguing Rules (AACR2) e o formato de registro bibliográfico Machine Readable Cataloging (MARC), conhecidos e aceitos internacionalmente. Além disso, o profissional da área pode recorrer ao compartilhamento ou captura de registros bibliográficos para facilitar seu trabalho por meio da catalogação cooperativa, bem como por meio dos catálogos online disponíveis por diferentes bibliotecas.

Os termos representação descritiva e representação temática, provavelmente, tenham sido adotados com o objetivo de ampliar o conceito de catalogação, o qual remete à produção de catálogos de biblioteca. Entretanto:

[...] o desenvolvimento teórico e metodológico da representação descritiva e da representação temática foi realizado de modo separado, conduzindo a dificuldades conceituais na articulação entre os dois processos. Já a Catalogação, incluindo a catalogação descritiva e a catalogação de assunto, responde pela construção global do sistema, nesse caso, o catálogo (Ortega, 2011, p.45).

A catalogação é o processo por meio do qual se descreve formalmente um documento ou recurso e se estabelece um variado e variável número de pontos de acesso, objetivando proporcionar, ao usuário final, a condição de encontrar, identificar, selecionar e obter o documento ou o recurso descrito, ou a informação nele contida. Portanto, é um processo que gera o registro bibliográfico que, por sua vez, identifica e descreve um documento, tanto na sua forma física, como na de conteúdo, funcionando como sua representação na medida em que permite a um usuário encontrar, identificar, selecionar e obter uma obra por meio de um instrumento de recuperação, nomeadamente o catálogo. Em outras palavras, uma catalogação bem elaborada permite poupar tempo, uma vez que evita a consulta a documentos não pertinentes e permite substituir a consulta a alguns documentos, pelo fato de o usuário já ter encontrado a informação desejada (Souza, 2009).

Naturalmente, bibliotecas cujos objetivos e usuários são diferentes não possuem as mesmas necessidades. Existe um elenco mínimo de dados capaz de identificar e individualizar um documento, a partir do qual qualquer Unidade de Informação pode definir as informações adicionais necessárias aos seus objetivos e aos seus usuários.

Considera-se, a partir do exposto, que a representação descritiva é uma parte fundamental no tratamento dos documentos ou recursos, ao permitir sua identificação dentro da memória documental.

No panorama atual, a representação descritiva vem sofrendo nos modelos convencionais de descrição bibliográfica, mudanças significativas que são provocadas pelas tecnologias de informação e comunicação, o que 
obrigatoriamente requer olhares diferenciados no tratamento dos recursos informacionais, inclusive a adoção de suas práticas na organização da informação na Web.

\section{Representação descritiva e a web semântica}

Como destacado anteriormente, o alicerce da Web Semântica é o RDF, que é uma forma de representação dos metadados sobre recursos Web (tais como título, autor, data de modificação de uma página da Web, copyright etc.) (Manola; Miller, 2004).

Lendo essa definição à luz dos conceitos apresentados relativos tanto à Web Semântica quanto à representação descritiva, fica clara a relação das atividades desenvolvidas em ambas as áreas.

Concorda-se com Mey $(1995$, p.6) quando afirma que "a riqueza da catalogação repousa nos relacionamentos entre os itens estabelecidos, de forma a criar alternativas de escolha para os usuários". Isso quer dizer que, se um usuário busca determinada obra, ele terá a oportunidade de optar por diferentes versões ou suportes, ou seja, as diferentes manifestações do mesmo item, como livro, dvd, CD-ROM, documento online, bem como em diferentes edições, traduções, línguas, e isso certamente ampliará o universo de pesquisa e de escolha desse usuário. Os relacionamentos entre os itens também lhe permitem localizar itens por ele desconhecidos, uma vez que a catalogação propicia a reunião de todos os itens sobre um determinado assunto, aumentando e melhorando a busca.

Enquanto Mey (1995) assevera que a riqueza da catalogação está nos relacionamentos entre itens, o fundamento da Web Semântica está nos dados lincados. Observa-se, então, que tanto as atividades da catalogação quanto as da Web Semântica preocupam-se em propiciar aos usuários formas mais ricas e amplas de recuperação da informação.

O uso de códigos normalizados na representação descritiva é fundamental por regularizar e padronizar procedimentos em sistemas de informação, especialmente de médio e grande porte, possibilitando maior consistência, eficiência e qualidade ao sistema, ao fornecer registros coerentes entre si; tal uso é fundamental também por viabilizar a cooperação catalográfica entre Unidades de Informação, diminuir a necessidade de tomar decisões individuais para cada documento a ser catalogado e facilitar os processos de informatização.

Mey (1995, p.39) observa que:

À descrição cabe extrair diretamente do item todas as informações, de interesse para o usuário, que individualizem o item tornando-o único entre os demais. Cada item possui apenas uma descrição e cada descrição se aplica a um único item.

As recomendações do W3C também objetivam a padronização de metadados de forma a tornar a descrição de cada item única, mas, ao mesmo tempo, formatada de modo a permitir que todos os dados fiquem acessíveis para que se criem vários produtos ou aplicações.

OW3C propõe que todos os dados e metadados constantes ou citados na Web possam ser processados por máquinas e para tanto sugere como fundamento o RDF, que é um modelo de dados para a descrição de recursos da Web. Portanto, a base da Web Semântica é a representação descritiva dos recursos a partir de um modelo em que os registros de metadados são representados por um conjunto de declarações formadas pelos já mencionados triplos (Subject/Predicate/Object).

No contexto tradicional da organização da informação nas Unidades de Informação (bibliotecas, arquivos, museus etc.), como os profissionais da informação poderão proceder para que os dados por eles processados, disponibilizados e recuperados possam ser processáveis por máquinas, conforme propõe o W3C?

Os tradicionais códigos de catalogação, vocabulários, tesauros, índices etc., deverão ser formatados dentro dos padrões propostos pelo W3C para a Web Semântica. No entanto, deve-se manter a adoção dos códigos de catalogação e das normas utilizadas para a descrição dos recursos bibliográficos. Contudo, tanto os pontos de acesso quanto os elementos de descrição deverão ser formatados no modelo RDF, ou seja, cada ponto de acesso e cada elemento de descrição devem ser representados em declarações RDF. Desta forma, os dados bibliográficos seriam legíveis por máquinas conforme recomendações do W3C. Esta constatação reforça o pressuposto de que a práxis da representação descritiva não só pode, como 
deve, ser uma contribuição para a Web Semântica, no sentido de que se mantenham características e funcionalidades das bases bibliográficas que vêm sendo aprimoradas há décadas.

Inicialmente devemos pensar em como estão organizados os bancos de dados bibliográficos atualmente. Estes bancos de dados contêm os registros bibliográficos, produtos da descrição, compostos de conjuntos de metadados, tais como o formato MARC (presente nos OPAC) e o Dublin Core adotado pela Open Arquive Initiative (OAI) como a base da interoperabilidade dos repositórios.

Coyle (2010c, p.15) argumenta que, apesar de o MARC estar estruturado em campos e subcampos que são legíveis por máquinas, tem sua base em linguagem textual, o que dificulta a aplicação das tecnologias da Web Semântica. Porém o fato de os dados bibliográficos serem fundamentalmente textuais não significa que sejam livres; estes possuem valores controlados, a exemplo do controle de autoridade e de cabeçalhos de assuntos.

Um outro componente importante para que possamos visualizar a relação entre a representação descritiva e a proposta de organização de recursos da Web Semântica são os novos desenvolvimentos da área: o Functional Requirements for Bibliographic Records (FRBR) desenvolvido pela International Federation of Library Associations (IFLA), é resultado de um estudo sobre os requisitos funcionais para registros bibliográficos empreendido de 1992 a 1997. O objetivo do estudo "era produzir uma estrutura (framework) que proporcionasse uma compreensão clara, definida com precisão e comumente compartilhada de como o registro bibliográfico pode proporcionar informação sobre o que se espera que o registro possa arquivar em termos de resposta às necessidades do usuário" (Le Boeuf, 2007, p.1) (um dos principais objetos de estudo, atualmente, na área da representação descritiva) e o Resource Description and Access (RDA) que é a proposta do novo padrão que visa fornecer um conjunto de diretrizes e instruções na descrição de recurso e acesso cobrindo todo tipo de conteúdo e mídia.

Supõe-se que para incluir os tradicionais catálogos no ambiente da Web Semântica, ou seja, transformar os dados bibliográficos em dados lincados na Web faz-se necessário modelar os dados já existentes que se utilizam de MARC ou Dublin Core, por exemplo, nos moldes do RDF. Deve-se, ainda, tornar o modelo conceitual FRBR e o código de catalogação RDA, recentemente publicado como sucessor do AACR, em base RDF; além de escrever os vocabulários controlados existentes em linguagens propostas pelo W3C (RDF e RDF Schema, simple Knowledge Organization System (SKOS), OWL e RIF). Neste sentido, várias ações já vêm sendo desenvolvidas. A seguir apresentaremos algumas ações identificadas nesta pesquisa.

Uma importante iniciativa é da Library of Congress (LC), que disponibiliza uma lista de cabeçalhos em URI (http://id.loc.gov/), ou seja, para cada cabeçalho, foi criado um URI, que é um identificador único (base para as declarações em RDF). Exemplo para o nome Jorge Amado: para Amado, Jorge, 1912-2001 a URl é <http://id.loc.gov/ authorities/names/n50024126>. Portanto, ao se desenvolverem aplicações de dados bibliográficos em RDF, pode-se utilizar o namespace dos URI criados pela LC.

Adaptando o exemplo sugerido por Coyle (2010c), pode-se dizer que para uma declaração em RDF do ponto de acesso "autor" para a obra Gabriela Cravo e Canela, poder-se-iam utilizar alguns identificadores já existentes. A seguir apresenta-se a declaração (Subject/Predicate) Object) com seus respectivos identificadores URI:

Subject: o livro (identificador do WordCat) http:// www.worldcat.org/title/gabriela-cravoe-canela/oclc /11427374

Predicate: a propriedade (identificador do RDAVocab) author - http://rdvocab.info/roles/ author

Object: o valor correspondente à propriedade (identificador da LC) - http://id.loc.gov/ authorities/names/n50024126

Esta iniciativa vem ao encontro do principal desafio da Web Semântica, que são os identificadores únicos para cada recurso. Segundo Coyle (2010c), para darmos visibilidade aos dados das bibliotecas na Web, primeiramente, é necessário que eles tenham identificadores na forma de URI. Neste sentido, vale destacar as atividades do Dublin Core Metadata Initiative (DCMI), organização responsável pela manutenção e desenvolvimento do conjunto de metadados Dublin Core, que é um 
conjunto de metadados criado para a descrição de recursos da Web e que é atualmente a base para a interoperabilidade dos repositórios digitais (temáticos ou institucionais).

A Dublin Core Metadata Initiative criou um identificador para cada um de seus elementos de metadados, disponíveis em <http://purl.org/dc/terms >. Cada um dos termos é definido de forma a especificar detalhadamente o seu uso. Para alguns termos, o DCMI define, inclusive, o tipo de valor que pode ser utilizado na descrição, como, por exemplo, para o metadado Date, sugere o uso da norma ISO 8601, que estabelece como descrever uma data (Coyle, 2010c). Os termos DCMI também estão representados em linguagem RDF Schema (Dublin Core Metadata Initiative, 2011).

Uma outra iniciativa DCMI é a publicação de um documento contendo recomendações para expressar os metadados Dublin Core em RDF (Nilsson et al., 2008) por meio de uma representação do modelo DCMI, ou seja, o Dublin Core Abstract Model (DCAM) em RDF.

O RDF também está presente no documento Guidelines for Dublin Core Application Profiles, que apresenta diretrizes para a criação de perfis de aplicação; descreve os componentes-chave; bem como delineia o processo de desenvolvimento de um perfil de aplicação Dublin Core (Coyle; Baker, 2008). O documento apresenta, ainda, o Singapore Framework, um modelo que relaciona os elementos descritivos de um perfil de aplicação (camada Application Profile) com os padrões de domínio (camada Domain Standards) e com as normas que fundamentam a Web Semântica (camada Foundation Standards), ou seja, a base para que um perfil de aplicação seja processável por máquina e, consequentemente, inserido na Web Semântica é o RDF e o RDFSchema (Figura 4).

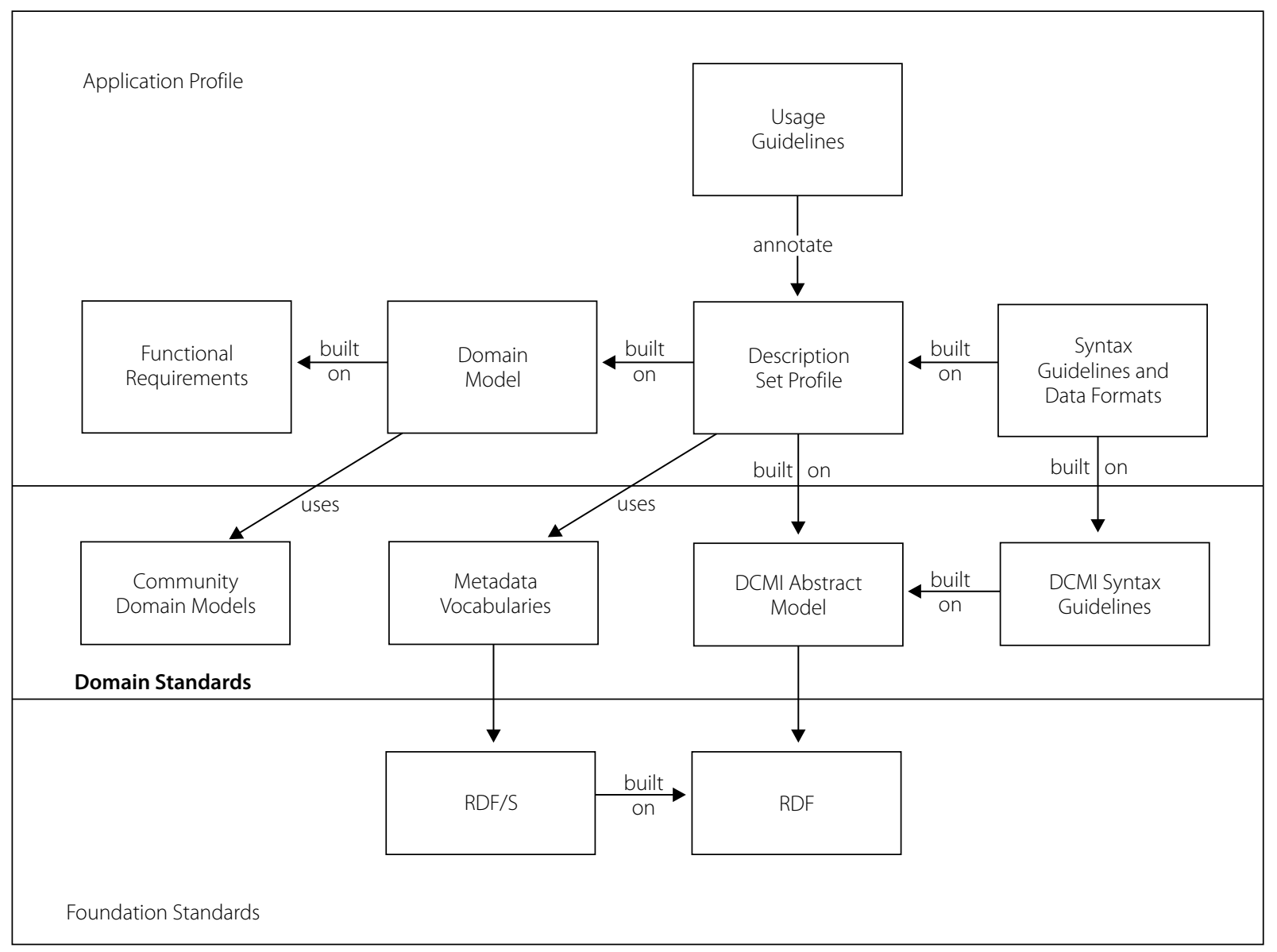

Figura 4. Singapore Framework.

Fonte: Coyle e Baker (2008). 
Relativamente ao RDA, vale destacar a iniciativa do DCMI em conjunto com o Joint Steering Committee (JSC). Num encontro promovido, em 2007, pela American Library Association (ALA) em conjunto com a British Library, os membros do DCMI e do JSC se dispuseram a criar uma expressão do RDA em RDF incluindo as associações deste com as entidades e relacionamentos do FRBR. O resultado é um registro online de RDA em RDF, que é a primeira definição de dados bibliográficos no formato para Web Semântica (Coyle, 2010a). Um artigo foi publicado na D-Lib Magazine relatando o processo de criação do RDA Vocabularies (Hillmann et al., 2010).

Na Figura 5, pode-se visualizar o detalhamento da propriedade Author (Work) apresentado no Open MetadataRegistry.

Em relação ao FRBR, existem críticas ao modelo, mas também algumas iniciativas para expressá-lo em RDF. Coyle (2010b, p.19, 2010d, p.25) cita:"expression of Core FRBRConcepts in RDF" (ver http://vocab.org/frbr/core.html) e o FRBRoo, que é parte do "CIDOC Conceptual Reference
Model" (ver http://cidoc.ics.forth.gr/frbr_inro. html). A primeira iniciativa é um vocabulário que inclui as classes de entidades dos grupos 1, 2 e 3 do FRBR, bem como as propriedades correspondentes para os relaciona-mentos entre essas entidades. A segunda, o FRBRoo, é uma ontologia criada para a captura e representação da semântica subjacente das informações bibliográficas e para facilitar a integração, mediação e intercâmbio de informações bibliográficas e de museus. Ambas consideram necessário incluir mais algumas entidades no modelo FRBR para poder formatá-lo adequadamente em RDF.

Pode-se considerar que as iniciativas acima descritas são importantíssimas e essenciais para inserir as bases bibliográficas na Web Semântica. No entanto há, ainda, muito a ser feito. Para a Ciência da Informação, torna-se um campo fértil para desenvolvimento de pesquisas que, inclusive, resultarão no aprimoramento da formação dos profissionais arquivistas, bibliotecários e museólogos, no sentido de estes serem capacitados para

Element Sets: RDA Roles

Elements: Author (Work)

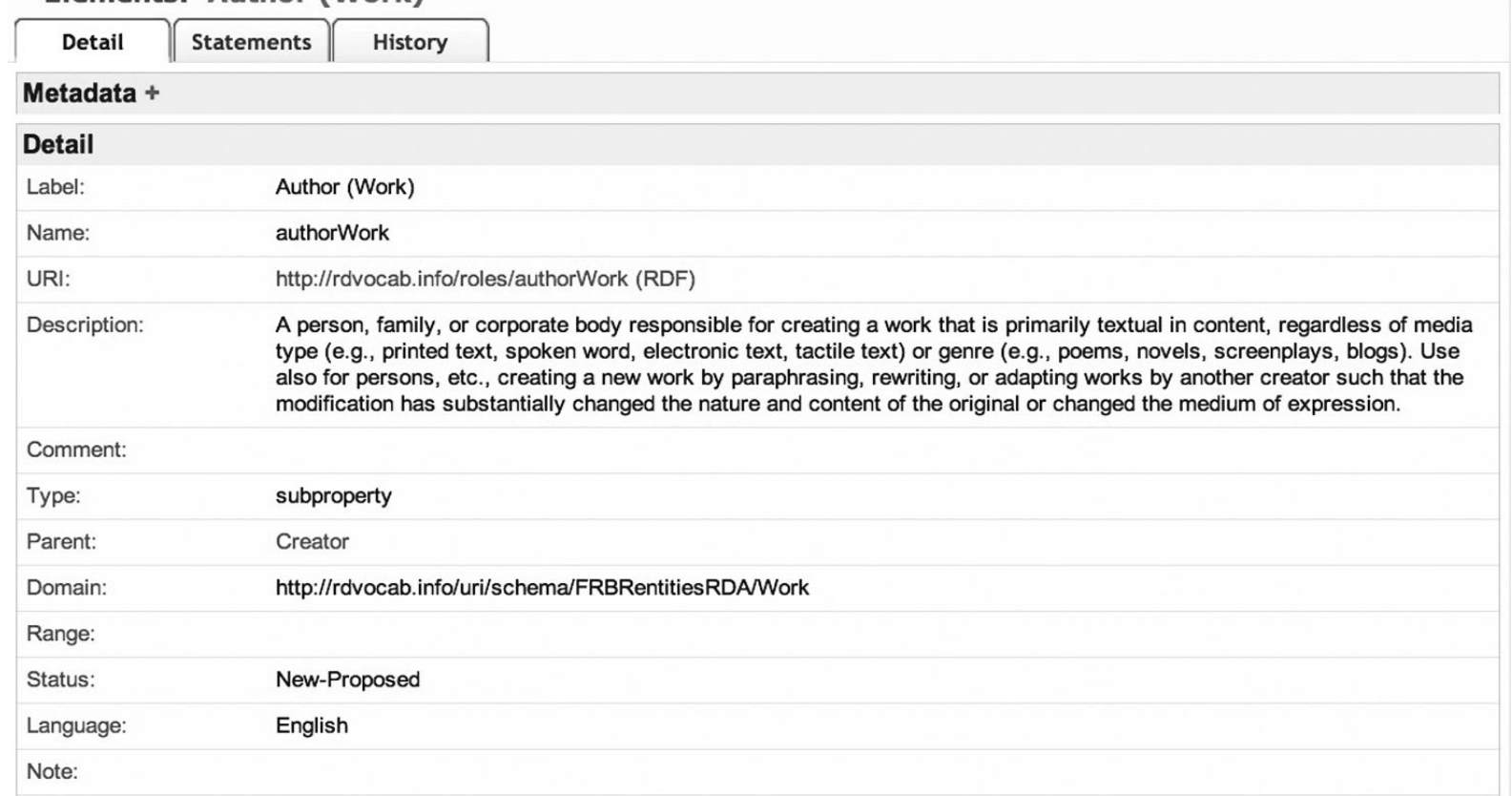

Figura 5. Propriedade Autor da Obra no Open Metadata Registry.

Fonte: <http://metadataregistry.org/schemaprop/show/id/121.html>. 
atuar na Web Semântica, ou seja, a aplicação dos conceitos e práticas da organização da informação na organização da informação da Web.

\section{Considerações Finais}

No que se refere ao objetivo deste estudo, pode-se concluir que as práticas da representação descritiva estão relacionadas com a proposta de organização dos recursos da Web, no contexto da Web Semântica. Esta constatação se deve ao fato de que, para que os dados bibliográficos contidos nos catálogos possam ser transformados em dados lincados da Web, é necessário que os catalogadores atuem na implantação do modelo de descrição RDF nos códigos e normas de catalogação vigentes atualmente.

A representação descritiva é uma ação que está presente no projeto da Web Semântica na medida em que a base para a existência deste projeto está no uso do RDF (um modelo de descrição). Portanto, a práxis do profissional da informação que cataloga recursos com base nos códigos e modelos que norteiam este fazer (ISBD, AACR, RDA, FRBR, MARC etc.) pode ser aplicada para colaborar com o projeto do W3C. A chave está na conversão dos dados bibliográficos atuais em descrições baseadas no modelo RDF.

Observou-se, ainda, que as ações que já vem sendo realizadas têm caminhado no sentido de permitir que os dados bibliográficos contidos nos catálogos bibliográficos possam ser inseridos em declarações nos moldes do RDF.

A transformação dos catálogos das bibliotecas e de outras bases de dados para dados lincados permitirá uma infinidade de aplicações. Os usuários poderão, ao consultar um catálogo, não somente obter informações a respeito de materiais bibliográficos (impressos ou

\section{Referências}

ADIDA, B.; BIRBECK, M. RDFa primer. Massachusetts: W3C, 2008. Available from: <http://www.w3.org/TR/xhtml-rdfaprimer>. Cited: 27 Apr. 2010.

ALLEMANG, D.; HENDLER, J. Semantic web for the working ontologist: effective modeling in RDFS and OWL. 2nd.ed. Amsterdan: Morgan Kaufmann, 2011. digitais) que estão contidos numa coleção específica; mas poderão, por exemplo, ao realizar uma busca, saber quais títulos relacionados existem na biblioteca local, em outras bibliotecas, em livreiros, em coleções particulares; obter acesso aos dados disponíveis no Google, Google Acadêmico, Open Library, Googlereader, Library Thing, entre outros; ser informados de grupos de discussão existentes sobre o tema de interesse; descobrir pesquisas em andamento; obter o ranking dos mais consultados etc.

Os catálogos poderão estar com seus metadados disponíveis por meio dos mecanismos de busca, e este seria o caminho contrário, ou seja, os usuários que estão realizando suas pesquisas nos mecanismos de busca seriam direcionados aos dados lincados das bibliotecas. Esta é uma ação essencial, pois é fato que a maioria dos usuários têm dado prioridade às buscas na Web, acessando cada vez menos os tradicionais catálogos para suprir suas necessidades informacionais.

Transformar os dados bibliográficos em dados lincados não enriquecerá apenas os serviços ofertados pelas bibliotecas aos seus usuários, mas, inclusive, a própria Web, na medida em que será possível, por meio dos mecanismos de busca, acessar os dados bibliográficos presentes nos catálogos de milhares de bibliotecas do mundo todo.

O resultado desta pesquisa não esgota o assunto, porém aponta para a real colaboração da representação descritiva para a Web Semântica, inclusive demonstrando que algumas organizações, tais como LC e DCMl, já estão realizando algumas ações iniciais. Portanto, é imprescindível que outros estudos sejam realizados, tanto por pesquisadores da área de Ciência da Informação (em especial a Organização da Informação) quanto do W3C, de forma a propor ações sistematizadas e globais para que os dados bibliográficos possam estar inseridos no conjunto dos dados lincados.

BERNERS-LEE, $T$ : HENDLER, J: LASSILA, O. The semantic web. Scientific American, v.284, n.5, p.34-43, 2001.

CATARINO, M.E. Integração das folksonomias aos metadados: identificação de novos elementos de metadados como contributo para a descrição de recursos em repositórios. 2009. Tese (Doutorado em Tecnologias e Sistemas de Infor- 
mação) - Departamento de Sistemas de Informação, Escola de Engenharia, Universidade do Minho, Minho, 2009.

COYLE, K. Library data in the web world. Library Technology Reports, v.46, n.2, p.5-11, 2010 a.

COYLE, K. Metadata models of the world wide web. Library Technology Reports, v.46, n.2, p.12-19, $2010 \mathrm{~b}$.

COYLE, K. RDA vocabularies for a twenty-first-century data. Library Technology Reports, v.46, n.1, p.14-29, 2010c.

COYLE, K. Understanding the semantic web: bibliographic data and metadata. Library Technology Reports, v.46, n.2, p.20-25, $2010 d$.

COYLE, K.; BAKER, T. Guidelines for Dublin Coreapplication profiles. 2008. Available from: <http://dublincore.org/documents/ 2008/11/03/profile-guidelines/>. Cited: 11 Oct. 2011.

DAS, S.; SUNDARA, S.; CYGANIAK, R. R2RML: RDB to RDF mapping language. Massachusetts:W3C, 2010. Available from: <http://www.w3.org/TR/2010/WD-r2rml-20101028/>. Cited: 10 Oct. 2011

DUBLIN CORE METADATA INITIATIVE. DCMI terms declarations represented in RDF schema language. 2011. Available from: <http://dublincore.org /schemas/rdfs>. Cited: 18 Oct. 2011.

HAHN, R. et al. Faceted wikipedia search. In: INTERNATIONAL CONFERENCE ON BUSINESS INFORMATION SYSTEMS, 13. 2010, Berlin. Proceedings electronics... Berlin: Springerlink, 2010. Available from: <http://www4.wiwiss.fu-berlin.de/ bizer/pub/hahn-etal-faceted-wikipedia-search-BIS2010.pdf>. Cited: 19 June 2011

HALPIN, H.; DAVIS, I. GRDDL primer. Massachusetts: W3C, 2007. Available from: <http://www.w3.org/TR/grddlprimer>. Cited: 8 July 2010.

HILLMANN, D. et al. RDA vocabularies. D-Lib Magazine, v.16, n.1-2, 2010. Available from: <http://www.dlib.org/dlib/ january10/hillmann/01 hillmann.html>. Cited: 20 Oct. 2011.

KING, R. Q\&A with Tim Berners-Lee. BusinessWeek, 2007. Available from: <http://www.ece.uc.edu/ mazlack/ECE. 716.Sp2010/Semantic.Web.Business.Week.2007/QA with_Tim_Berners_Lee.pdf>. Cited: 8 July 2010.

LE BOEUF, P. O admirável mundo novo do FRBR: versão 5. 2007. Disponível em: <http://www.imeicc5.com/download/ portuguese/Presentations2C_BraveNewFRBRWorld(PR)_ Port.pdf>. Acesso em: 9 out. 2011.

MANOLA, F.; MILLER, E. RDF primer. 2004. Available from: <http://www.w3.org/TR/2004/REC-rdf-primer-20040210/>. Cited: 1 Sept. 2010.
MEY, E.S.A. Introdução à catalogação. Brasília: Briquet de Lemos, 1995.

MORGENSTERN, L.; WELTY, C.; BOLEY, H. RIF primer. 2010. Available from: <http://www.w3.org/2005/rules/wiki/ Primer>. Cited: 14 Oct. 2011.

MURCHO, D. Dicionário escolar de filosofia. Lisboa: Plátano, 2011. Disponível em: <http://www.defnarede.com/i.html>. Acesso em: 10 out. 2011.

NILSSON, M. et al. Expressing Dublin Core metadata using the resource description framework. Singapore: DCMI, 2008. Available from: <http://dublincore.org/documents/dc-rdf $>$. Cited: 14 Oct. 2011.

ORTEGA, C.D. Do princípio monográfico à unidade documentária: exploração dos fundamentos da catalogação. Liinc em Revista, v.7, n.1, p.43-60, 2011.

PRUD'HOMMEAUX, E.; SEABORNE, A. SPARQL query language for RDF. Sverige: W3C, 2008. Available from: <http://www w3.org/TR/rdf-sparql-query>. Cited: 19 Oct. 2011.

RDF WORKING GROUP. Resource description framework. Massachusetts: W3C, 2004. Available from: <http://www.w3. org/RDF/>. Cited: 10 Oct. 2011.

SAWAYA, M.R. Dicionário de informática e internet. São Paulo: Nobel, 1999. Disponível em: <http://pt.scribd.com/doc/ 2989340/Informatica-Dicionario-Internet>. Acesso em: 16 fev. 2012.

SCHEPPE, K. Protocol for web description resources (powder): primer. 2009. Available from: <http://www.w3.org/TR/2009/ NOTE-powder-primer-20090901/>. Cited: 8 July 2010.

SOUZA, T.B. O ensino de representação descritiva nos cursos da área de ciência da informação no Brasil e em Portugal: estudo comparativo. 2009. Tese (Doutorado em Ciências Documentais) - Faculdade de Letras, Universidade do Porto, Porto, 2009.

WORD WIDE WEB CONSORTIUM. W3C semantic web activity. 2001. Available from: <http://www.w3.org/2001/sw/>. Cited: 12 Feb. 2008.

WORD WIDE WEB CONSORTIUM. W3C glossary dictionary. 2003. Available from: <http://www.w3.org/2003/glossary/ subglossary/REC-xml-names.rdf/>. Cited: 10 Oct. 2011.

WORD WIDE WEB CONSORTIUM. Semantic web. 2010. Available from: <http://www.w3.org/standards/semantic Web/>. Cited: 1 Sept. 2010.

WORD WIDE WEB CONSORTIUM. Semantic web terminology. 2011. Available from: <http://www.w3.org/2001/sw/wiki/ Semantic_Web_terminology>. Cited: 10 Oct. 2011. 